\title{
Supporting Information for \\ Attribution of lake warming in four shallow lakes in the Middle and Lower Yangtze River basin
}

\author{
Xinyu $\mathrm{Li}^{1,2}$, Shushi Peng ${ }^{1 *}$, Xuwei Deng ${ }^{3}$, Ming Su${ }^{4}$ and Hui Zeng ${ }^{2}$ \\ ${ }^{1}$ Sino-French Institute for Earth System Science, College of Urban and Environmental \\ Sciences, and Laboratory for Earth Surface Processes, Peking University, Beijing 100871, \\ China \\ ${ }^{2}$ Peking University Shenzhen Graduate School, Shenzhen 518055, China \\ ${ }^{3}$ Donghu Experimental Station of Lake Ecosystems, Institute of Hydrobiology, Chinese \\ Academy of Sciences, Wuhan 430072, China \\ ${ }^{4}$ Key Laboratory of Drinking Water Science and Technology, Research Center for Eco- \\ Environmental Sciences, Chinese Academy of Sciences, Beijing 100085, China \\ *Correspondence: Dr. Shushi Peng, Email: speng@pku.edu.cn
}

SI includes: 15 total pages, 8 figures and 6 tables. 


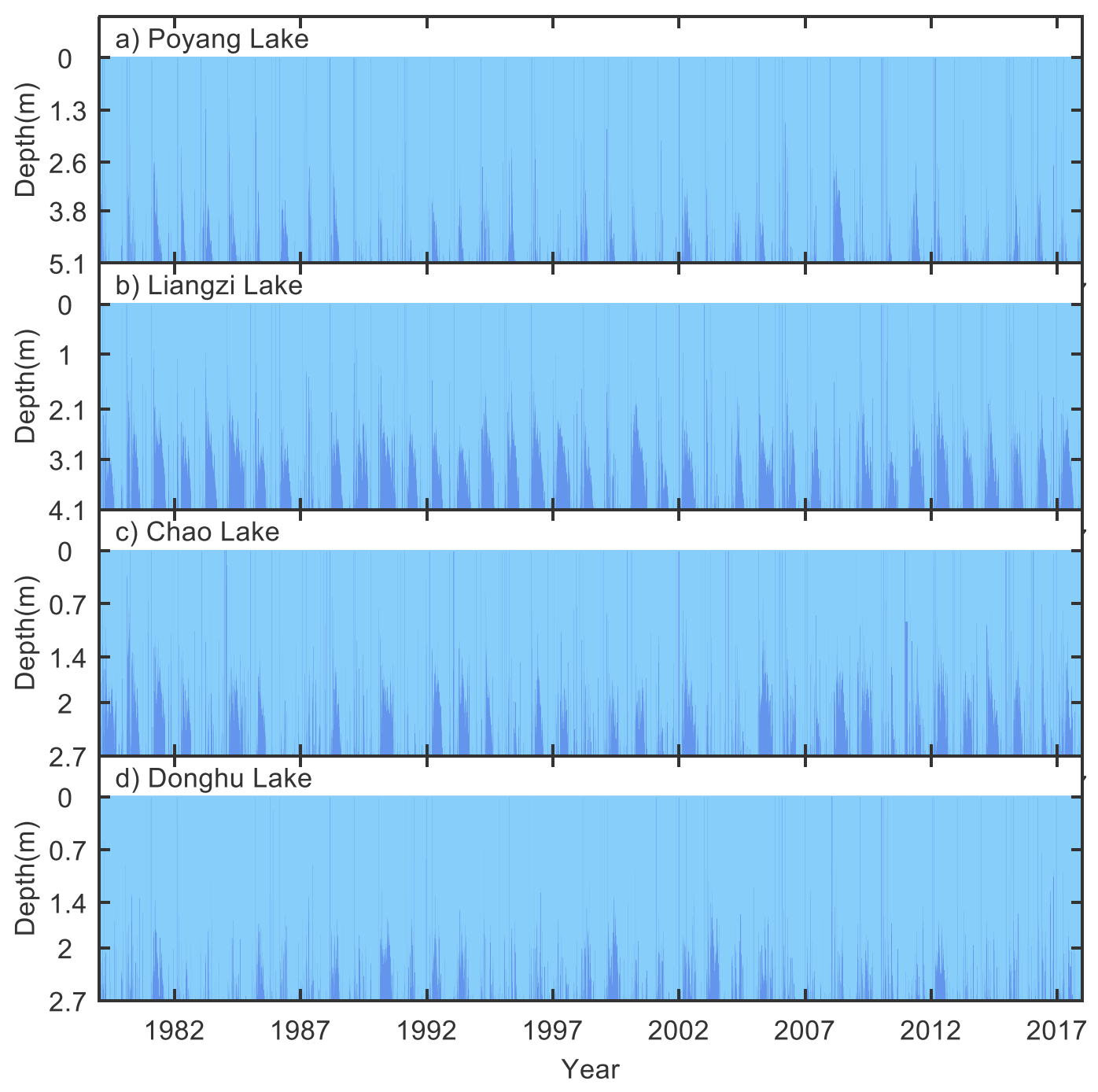

Figure S1. Lake stratification profile in four lakes during the period 1979-2017. Light blue areas indicate the depth of the mixed layer and dark blue areas indicate the depth of the thermocline, both of which are derived from the daily outputs driven by the FLake model. Liangzi Lake and Chao Lake have stratification phenomenon, while little stratification in Poyang Lake and Donghu Lake. 

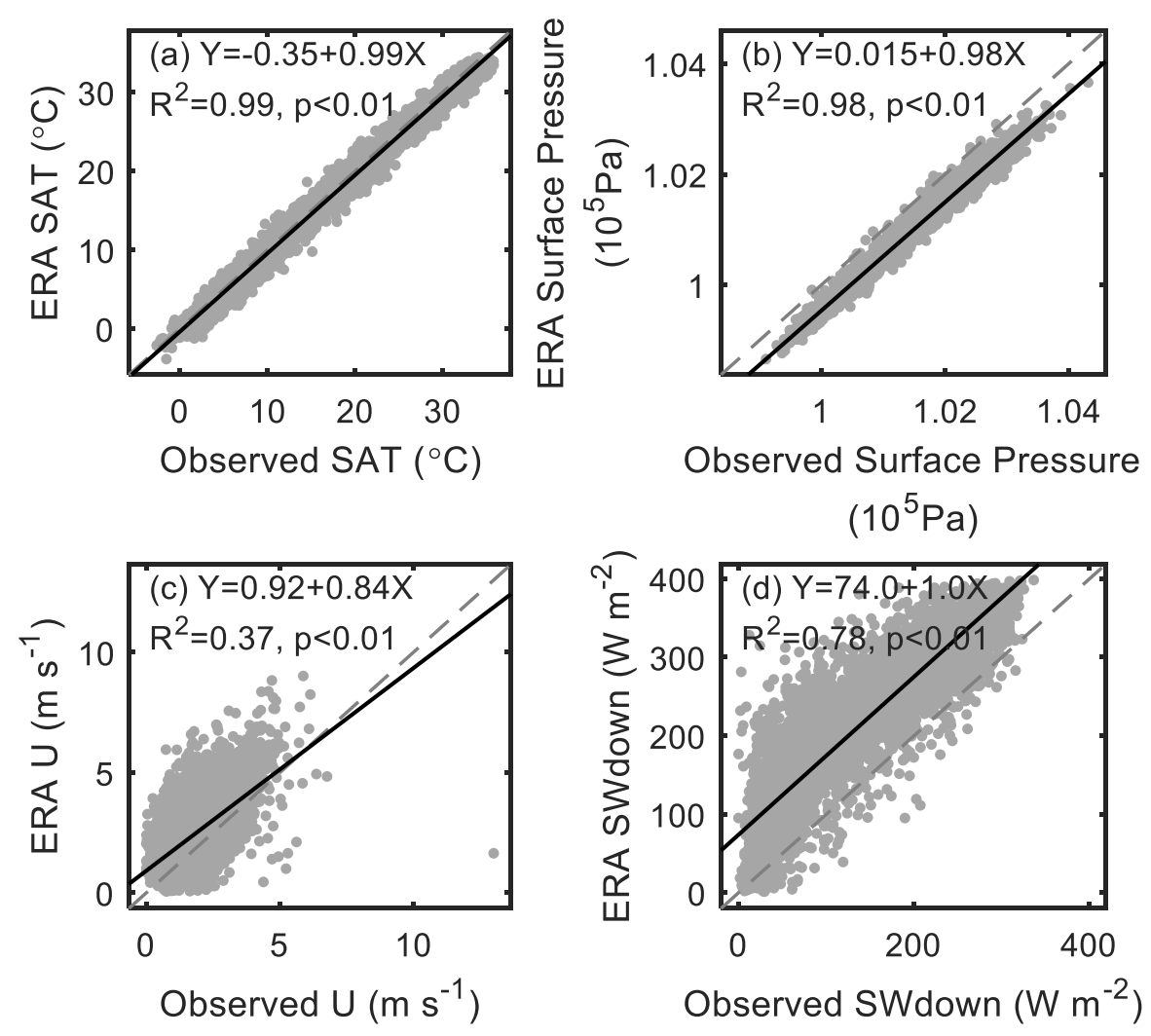

Figure S2. Comparison of daily (a) SAT, (b) Surface Pressure, (c) U and (d) SWdown between ERA-Interim and in situ data at Donghu Lake. 

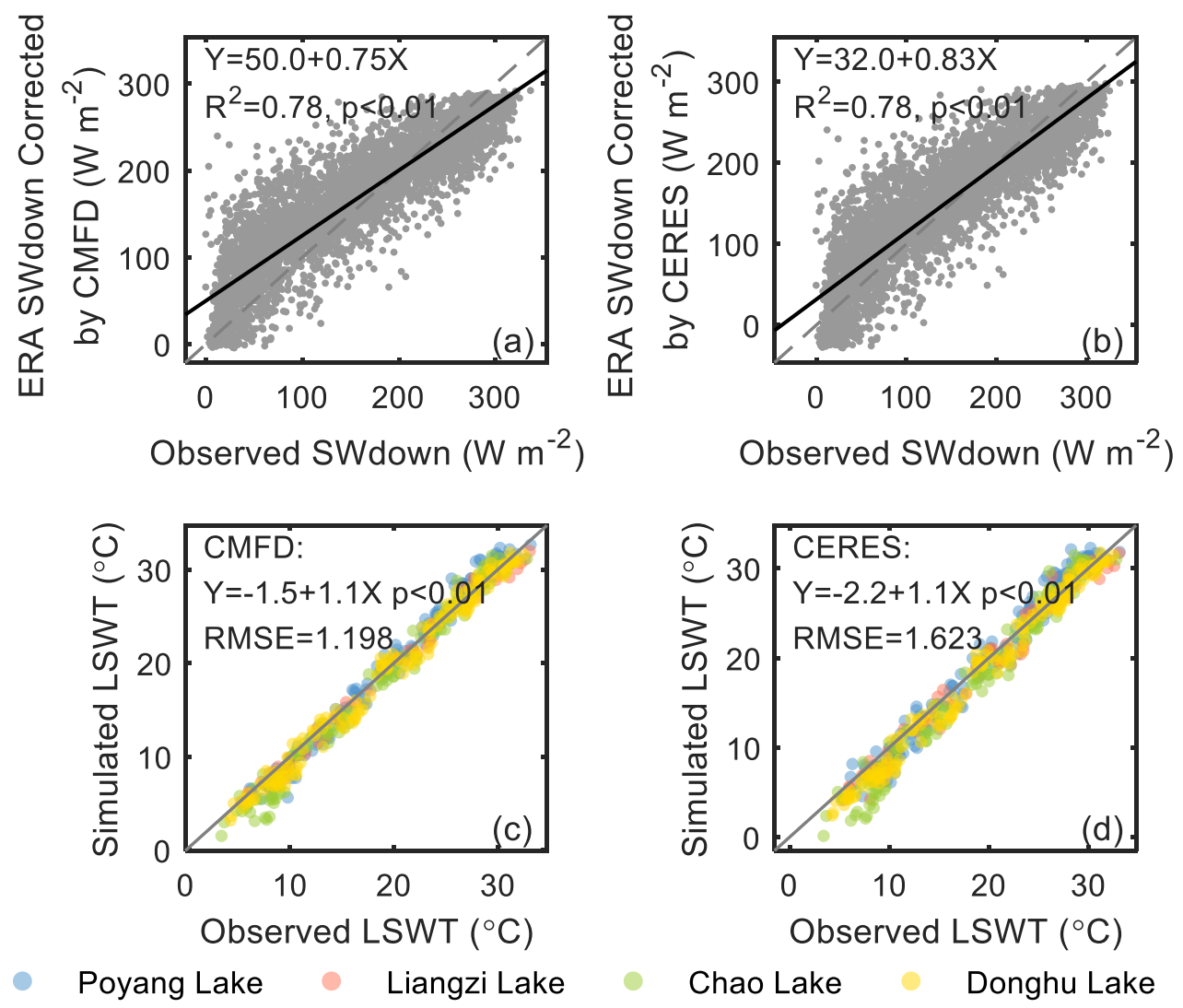

Figure S3. Comparison of daily SWdown (a) between in situ observations and ERA-Interim corrected by CMFD at the four lakes, and (b) between in situ observations and ERA-Interim corrected by CERES. Comparison of monthly LSWT (c) between in situ observations and simulations driven by SWdown from ERA-Interim corrected by CMFD, and (d) between in situ observations and simulations driven by SWdown from ERA-Interim corrected by CERES. 
- Spring - Summer - Autumn - Winter

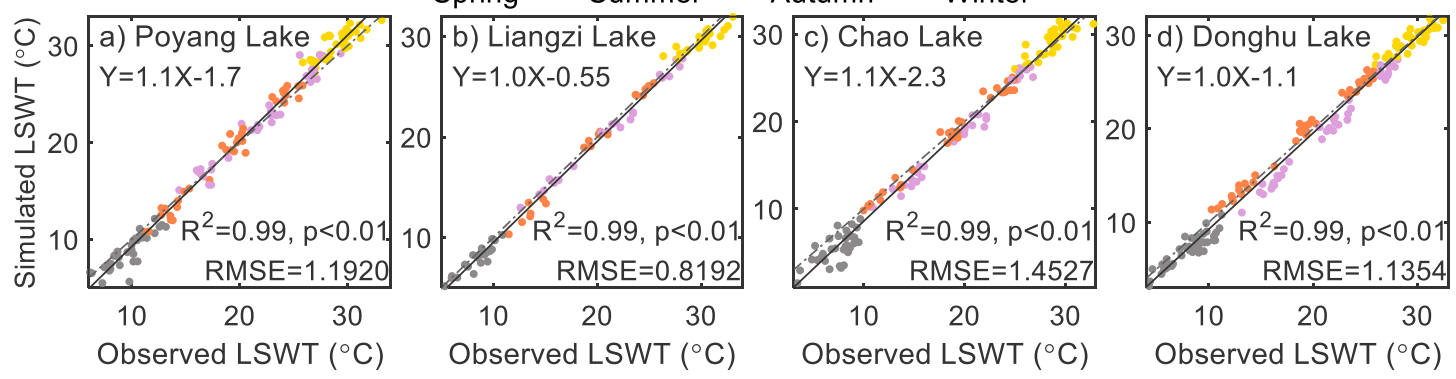

Figure S4. Comparison between monthly simulated and observed LSWT at the four lakes. The four colors indicate the four seasons respectively. 


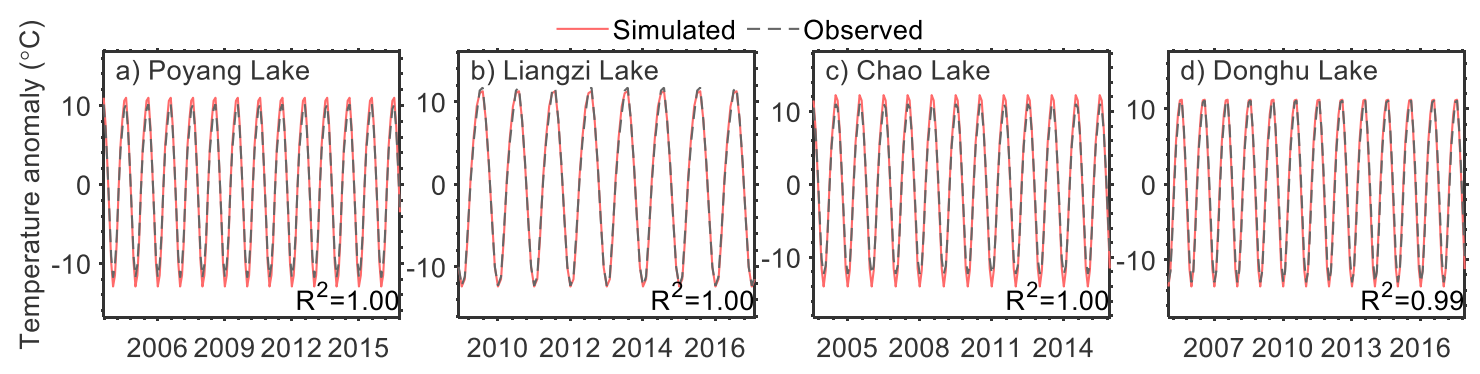

Figure S5. Comparison between the detrended climatological seasonality of monthly simulated (red line) and observed (black dots) LSWT at (a) Poyang Lake, (b) Liangzi Lake (c) Chao Lake and (d) Donghu Lake. 

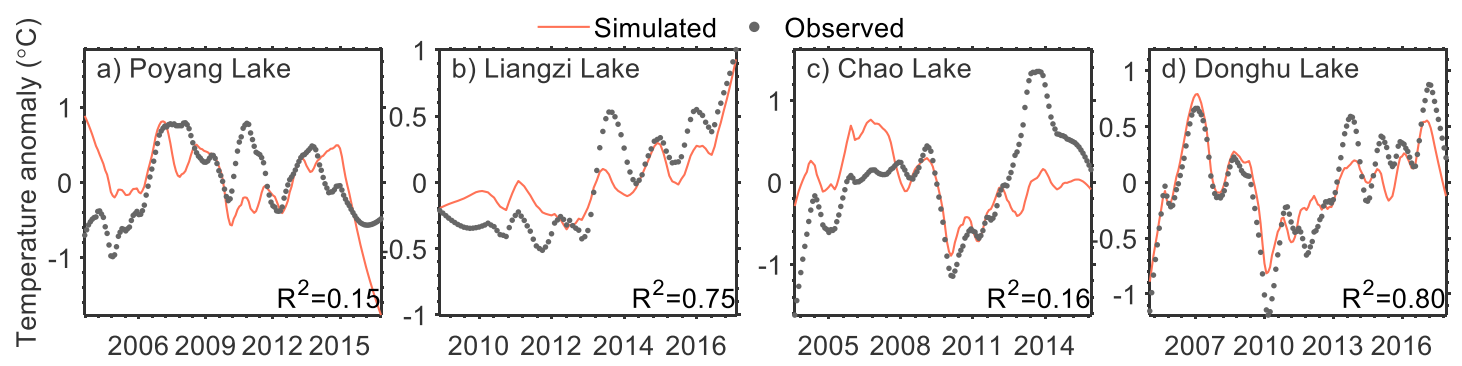

Figure S6. Comparison between de-seasonalized simulated (red line) and observed (black dots) LSWT trend at (a) Poyang Lake, (b) Liangzi Lake (c) Chao Lake and (d) Donghu Lake. 
a) Poyang Lake

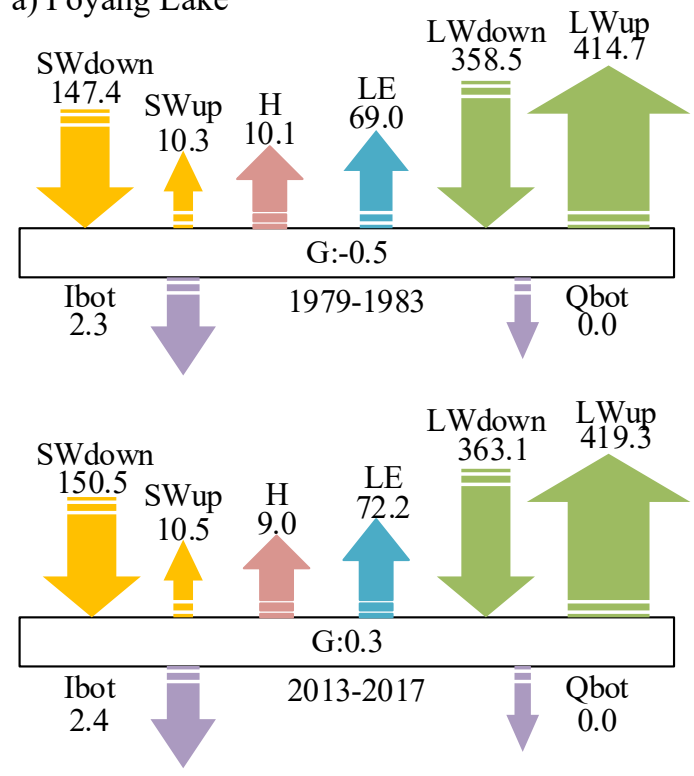

c) Chao Lake

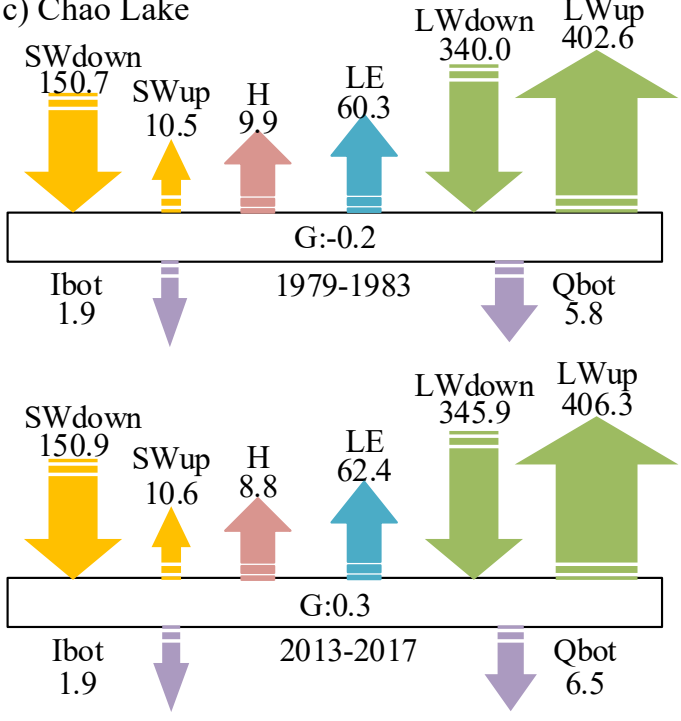

b) Liangzi Lake
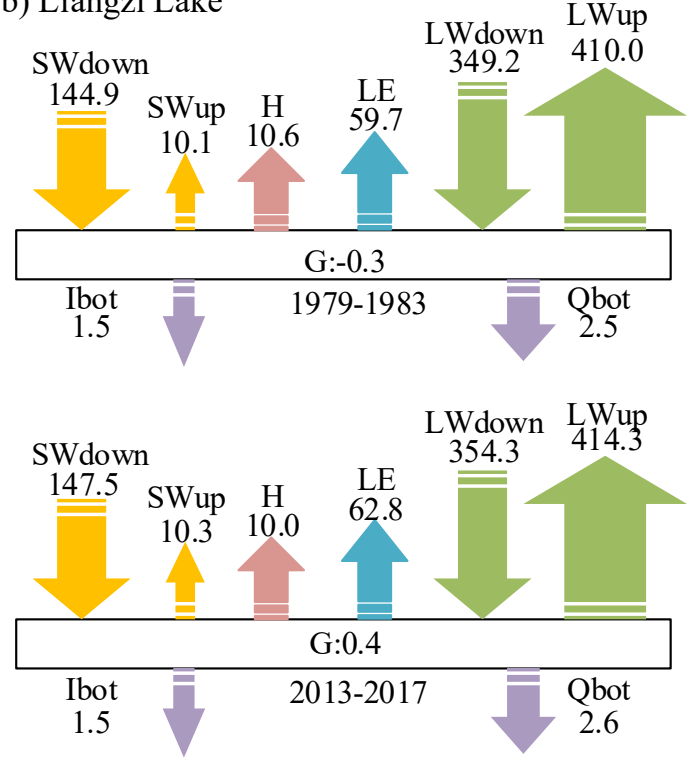

d) Donghu Lake

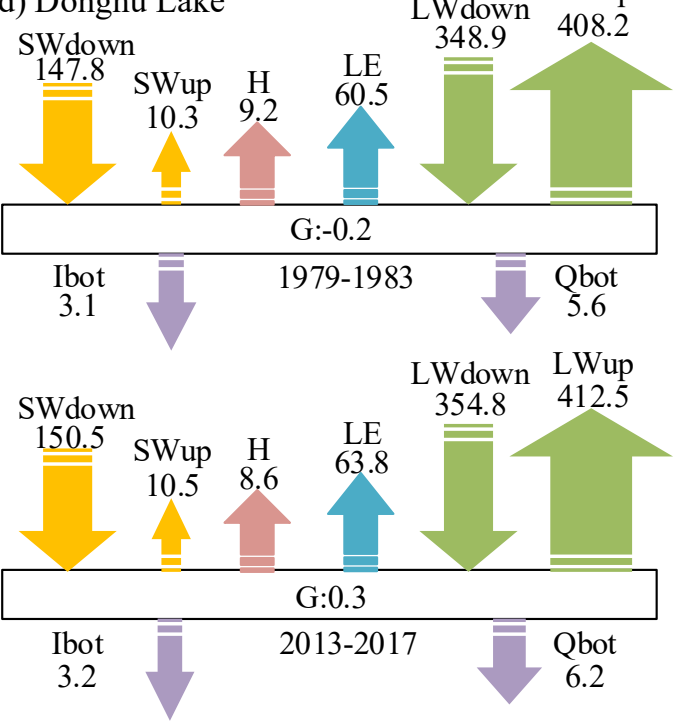

Figure S7. Same as Figure 4 but this figure shows the surface heat budgets at the four lakes. 


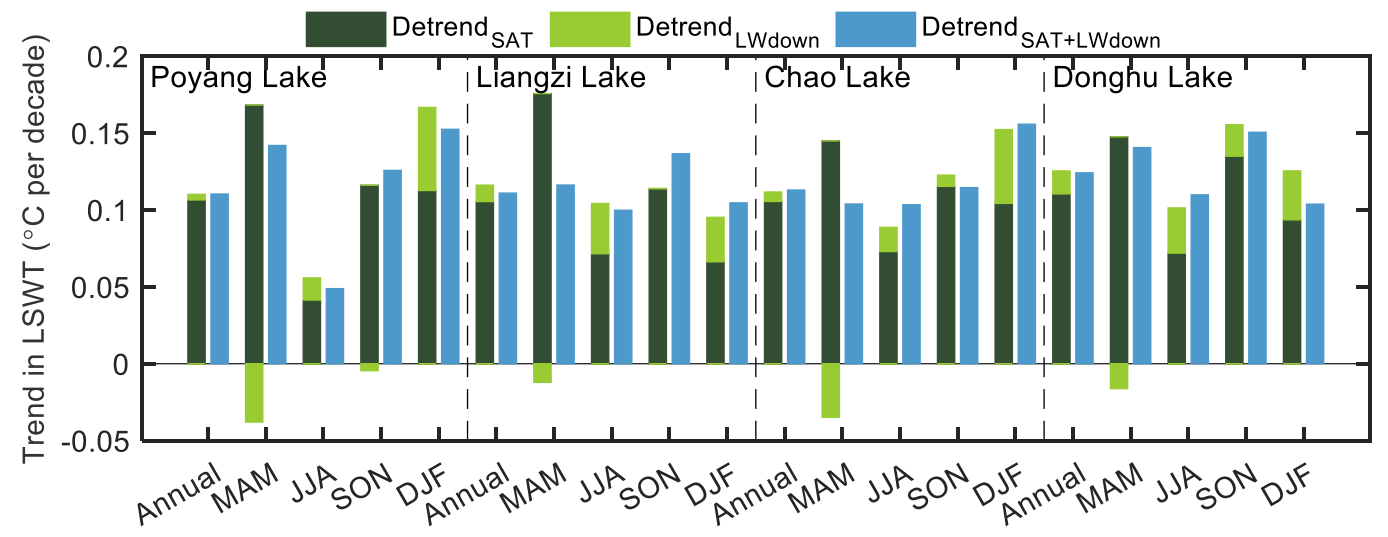

Figure S8. Interaction between LWdown and SAT on the trend in annual and seasonal (MAM, JJA, SON, DJF) LSWT from 1979 to 2017 for the four lakes. Detrend $d_{\text {SAT }}$ and Detrend ${ }_{\text {LWdown }}$ indicate the contributions of SAT and LWdown to LSWT warming by removing the trend of SAT and LWdown respectively (by S2 and S4 simulations). Detrend ${ }_{S A T+L W d o w n}$ indicates the contribution of SAT and LWdown to LSWT warming by removing the trend of both SAT and LWdown together. The difference between Detrend $d_{S A T}+$ Detrend $_{L W d o w n}$ and Detrend ${ }_{S A T+L W d o w n}$ shows the interaction between LWdown and SAT on LSWT warming. 
Table S1. Annual and seasonal mean depth of the mixed layer and total number of stratified days at Poyang Lake, Liangzi Lake, Chao Lake and Donghu Lake.

\begin{tabular}{cccccc}
\hline & \multicolumn{5}{c}{ Poyang Lake (depth=5.1m) } \\
\cline { 2 - 6 } & Annual & Spring & Summer & Autumn & Winter \\
\hline Depth of Mix Layer (m) & 4.63 & 4.28 & 4.38 & 5.05 & 4.80 \\
Number of stratified days (day) & 78 & 19 & 57 & 0 & 2 \\
\hline & \multicolumn{5}{c}{ Liangzi Lake (depth =4.1m) } \\
\cline { 2 - 6 } & Annual & Spring & Summer & Autumn & Winter \\
\hline Depth of Mix Layer (m) & 3.56 & 3.27 & 3.10 & 4.00 & 3.88 \\
Number of stratified days (day) & 120 & 36 & 82 & 0 & 2 \\
\hline & \multicolumn{5}{c}{ Chao Lake (depth $=2.7 \mathrm{~m})$} \\
\cline { 2 - 6 } & Annual & Spring & Summer & Autumn & Winter \\
\hline Depth of Mix Layer (m) & 2.41 & 2.28 & 2.27 & 2.64 & 2.47 \\
Number of stratified days (day) & 63 & 18 & 41 & 0 & 4 \\
\hline & \multicolumn{5}{c}{ Donghu Lake (depth $=2.7 \mathrm{~m})$} \\
\cline { 2 - 6 } & Annual & Spring & Summer & Autumn & Winter \\
\hline Depth of Mix Layer (m) & 2.59 & 2.54 & 2.64 & 2.65 & 2.54 \\
Number of stratified days (day) & 4 & 1 & 0 & 0 & 3 \\
\hline
\end{tabular}


Table S2. Key input parameters and general characteristics for each lake.

\begin{tabular}{|c|c|c|c|c|c|c|c|}
\hline $\begin{array}{l}\text { Lake } \\
\text { name }\end{array}$ & $\begin{array}{l}\text { Lake } \\
\text { depth } \\
\text { (m) }\end{array}$ & $\begin{array}{l}\text { Depth of the } \\
\text { thermally } \\
\text { active layer } \\
\text { of bottom } \\
\text { sediments } \\
\text { (m) }\end{array}$ & $\begin{array}{l}\text { Typical } \\
\text { wind } \\
\text { fetch } \\
\text { (m) }\end{array}$ & $\begin{array}{l}\text { Spinup } \\
\text { equilibrium } \\
\text { temperature of } \\
\text { the upper } \\
\text { mixed layer } \\
\left({ }^{\circ} \mathrm{C}\right)\end{array}$ & $\begin{array}{c}\text { Spinup } \\
\text { equilibrium } \\
\text { temperature at } \\
\text { the bottom } \\
\left({ }^{\circ} \mathrm{C}\right)\end{array}$ & $\begin{array}{l}\text { Spinup } \\
\text { equilibrium } \\
\text { temperature of } \\
\text { the thermally } \\
\text { active layer of } \\
\text { the bottom } \\
\text { sediments }\left({ }^{\circ} \mathrm{C}\right)\end{array}$ & $\begin{array}{l}\text { Extinction } \\
\text { coefficients } \\
\left(\mathrm{m}^{-1}\right)\end{array}$ \\
\hline $\begin{array}{c}\text { Poyang } \\
\text { Lake }\end{array}$ & 5.1 & 4.08 & 100 & 10.8 & 10.2 & 3.9 & 0.8 \\
\hline $\begin{array}{l}\text { Liang- } \\
\text { zi Lake }\end{array}$ & 4.1 & 3.28 & 100 & 8.2 & 8.2 & 5.4 & 1.1 \\
\hline $\begin{array}{l}\text { Chao } \\
\text { Lake }\end{array}$ & 2.7 & 2.16 & 100 & 5.4 & 5.4 & 3.8 & 1.6 \\
\hline $\begin{array}{c}\text { Donghu } \\
\text { Lake }\end{array}$ & 2.7 & 2.16 & 100 & 7.2 & 7.2 & 5.3 & 1.4 \\
\hline
\end{tabular}


Table S3. Simulation protocol in this study.

\begin{tabular}{ccccccc}
\hline Simulation & SAT & SWdown & LWdown & U & SH & SP \\
\hline S1 & $1979-2017$ & $1979-2017$ & $1979-2017$ & $1979-2017$ & $1979-2017$ & $1979-2017$ \\
S2 & 1979 & $1979-2017$ & $1979-2017$ & $1979-2017$ & $1979-2017$ & $1979-2017$ \\
S3 & $1979-2017$ & 1979 & $1979-2017$ & $1979-2017$ & $1979-2017$ & $1979-2017$ \\
S4 & $1979-2017$ & $1979-2017$ & 1979 & $1979-2017$ & $1979-2017$ & $1979-2017$ \\
S5 & $1979-2017$ & $1979-2017$ & $1979-2017$ & 1979 & $1979-2017$ & $1979-2017$ \\
S6 & $1979-2017$ & $1979-2017$ & $1979-2017$ & $1979-2017$ & 1979 & $1979-2017$ \\
S7 & $1979-2017$ & $1979-2017$ & $1979-2017$ & $1979-2017$ & $1979-2017$ & 1979 \\
\hline
\end{tabular}


Table S4. Contributions of different climate variables to the LSWT warming with their $95 \%$ confidence intervals.

\begin{tabular}{|c|c|c|c|c|c|}
\hline & Annual & Spring & Summer & Autumn & Winter \\
\hline & \multicolumn{5}{|c|}{$\left({ }^{\circ} \mathrm{C}\right.$ per decade $)$} \\
\hline & \multicolumn{5}{|c|}{ Poyang Lake } \\
\hline SAT & 0 & $0.17 \pm$ & $0.04 \pm 0.05$ & $0.12 \pm 0.06$ & 0.10 \\
\hline SWdown & $0.13 \pm 0.08$ & $0.31 \pm 0.16$ & $0.04 \pm 0.11$ & $0.13 \pm 0.11$ & $0.04 \pm 0.14$ \\
\hline LWdo & $0.00 \pm 0.05$ & $-0.04 \pm 0.11$ & $0.01 \pm 0.04$ & $0.00 \pm 0.09$ & $0.05 \pm 0.14$ \\
\hline $\mathrm{U}$ & $0.01 \pm 0.04$ & $0.01 \pm 0.09$ & $0.04 \pm 0.07$ & $0.02 \pm 0.08$ & $-0.01 \pm 0.06$ \\
\hline \multirow[t]{2}{*}{$\mathrm{SH}$} & $0.03 \pm 0.04$ & $0.09 \pm 0.09$ & -0.02 & $0.02 \pm 0.08$ & $0.01 \pm 0.10$ \\
\hline & \multicolumn{5}{|c|}{ Liangzi Lake } \\
\hline SAT & $0.11 \pm 0.04$ & $0.18 \pm 0.10$ & $0.07 \pm 0.06$ & $0.11 \pm 0.06$ & $.07 \pm 0.09$ \\
\hline SWdow & $0.15 \pm 0.08$ & $0.28 \pm 0.18$ & $0.10 \pm 0.10$ & $0.16 \pm 0.13$ & $0.07 \pm 0.12$ \\
\hline LWdown & $0.01 \pm 0.05$ & $-0.01 \pm 0.12$ & $0.03 \pm 0.03$ & $0.00 \pm 0.10$ & $0.03 \pm 0.15$ \\
\hline $\mathrm{U}$ & $0.00 \pm 0.04$ & $0.01 \pm 0.08$ & $-0.01 \pm 0.07$ & $0.02 \pm 0.07$ & $-0.02 \pm 0.05$ \\
\hline \multirow[t]{2}{*}{$\mathrm{SH}$} & $0.03 \pm 0.03$ & $0.09 \pm 0.07$ & $0.01 \pm 0.05$ & $0.01 \pm 0.07$ & $0.00 \pm 0.09$ \\
\hline & \multicolumn{5}{|c|}{ Chao Lake } \\
\hline SAT & $0.11 \pm 0.05$ & $0.14 \pm 0.09$ & $0.07 \pm 0.08$ & $0.12 \pm 0.06$ & $0.10 \pm 0.10$ \\
\hline SWdow & $0.12 \pm 0.07$ & $0.20 \pm 0.16$ & $0.08 \pm 0.12$ & $0.14 \pm 0.13$ & $0.06 \pm 0.15$ \\
\hline LWdow & $0.01 \pm 0.06$ & $-0.03 \pm 0.09$ & $0.02 \pm 0.05$ & $0.01 \pm 0.12$ & $0.05 \pm 0.16$ \\
\hline $\mathrm{U}$ & $0.00 \pm 0.03$ & $0.00 \pm 0.07$ & $0.02 \pm 0.06$ & $0.00 \pm 0.06$ & $-0.02 \pm 0.06$ \\
\hline \multirow[t]{2}{*}{$\mathrm{SH}$} & $0.02 \pm 0.04$ & $0.01 \pm 0.08$ & $0.02 \pm 0.06$ & $0.01 \pm 0.07$ & $0.02 \pm 0.09$ \\
\hline & \multicolumn{5}{|c|}{ Donghu Lake } \\
\hline SAT & $0.11 \pm 0.04$ & $0.15 \pm 0.09$ & $0.07 \pm 0.06$ & $0.13 \pm 0.06$ & $0.09 \pm 0.11$ \\
\hline SWdown & $0.12 \pm 0.07$ & $0.16 \pm 0.15$ & $0.12 \pm 0.10$ & $0.16 \pm 0.12$ & $0.09 \pm 0.12$ \\
\hline LWdov & $0.01 \pm 0.05$ & $-0.02 \pm 0.08$ & $0.03 \pm 0.04$ & $0.02 \pm 0.09$ & $0.03 \pm 0.14$ \\
\hline $\mathrm{U}$ & $-0.01 \pm 0.04$ & $0.00 \pm 0.07$ & $-0.04 \pm 0.08$ & $0.01 \pm 0.07$ & $-0.01 \pm 0.05$ \\
\hline $\mathrm{SH}$ & $0.01 \pm 0.04$ & $0.03 \pm 0.06$ & $0.00 \pm 0.04$ & $0.00 \pm 0.08$ & $0.02 \pm 0.08$ \\
\hline
\end{tabular}


Table S5. Trends of annual and seasonal climate forcing variables at the four lakes from 1979 to 2017. * indicates a trend with statistical significance $(p<0.05)$.

\begin{tabular}{|c|c|c|c|c|c|c|}
\hline \multirow{2}{*}{ Lake } & & SAT & \multicolumn{2}{|c|}{ SWdown LWdown } & $\mathrm{U}$ & $\mathrm{SH}$ \\
\hline & & \multicolumn{4}{|c|}{${ }^{\circ} \mathrm{C}$ per decade $\underset{\text { decade }}{\mathrm{W} \mathrm{m}^{-2} \text { per } \mathrm{W} \mathrm{m}^{-2} \text { per }}$ decade $\mathrm{m} \mathrm{s}^{-1}$ per decade } & per decade \\
\hline \multirow{5}{*}{ Poyang Lake } & Annual & $0.37 *$ & 2.17 & 0.88 & 0.007 & 0.0001 \\
\hline & Spring & $0.51 *$ & $6.28 *$ & -0.34 & -0.005 & 0.0001 \\
\hline & Summer & 0.16 & 0.17 & 0.91 & -0.017 & 0.0001 \\
\hline & Autumn & $0.47 *$ & 1.18 & 2.31 & 0.022 & 0.0001 \\
\hline & Winner & $0.42 *$ & 1.33 & 0.88 & 0.025 & 0.0001 \\
\hline \multirow{5}{*}{ Liangzi Lake } & Annual & $0.36^{*}$ & 2.05 & 1.00 & 0.006 & 0.0001 \\
\hline & Spring & $0.54 *$ & 4.77 & 0.53 & 0.003 & 0.0001 \\
\hline & Summer & 0.23 & 1.45 & 1.12 & 0.010 & 0.0001 \\
\hline & Autumn & $0.38 *$ & 1.31 & 2.04 & -0.004 & 0.0001 \\
\hline & Winner & 0.32 & 1.02 & 0.43 & 0.018 & 0.0000 \\
\hline \multirow{5}{*}{ Chao Lake } & Annual & $0.39 *$ & 1.60 & 1.12 & -0.003 & 0.0001 \\
\hline & Spring & $0.58 *$ & 4.25 & 0.34 & 0.002 & 0.0000 \\
\hline & Summer & 0.28 & 1.23 & $1.53 *$ & -0.027 & 0.0001 \\
\hline & Autumn & $0.38 *$ & 0.74 & 1.96 & 0.012 & 0.0001 \\
\hline & Winner & $0.36^{*}$ & 0.36 & 0.86 & 0.005 & 0.0000 \\
\hline \multirow{5}{*}{ Donghu Lake } & Annual & $0.37 *$ & 2.03 & $1.29 *$ & 0.008 & 0.0001 \\
\hline & Spring & $0.56^{*}$ & 4.51 & 0.86 & -0.003 & 0.0001 \\
\hline & Summer & 0.25 & 1.84 & 1.42 & 0.020 & 0.0001 \\
\hline & Autumn & $0.38 *$ & 1.20 & 2.36 & 0.002 & 0.0001 \\
\hline & Winner & 0.32 & 0.84 & 0.63 & 0.020 & 0.0000 \\
\hline
\end{tabular}


Table S6. The RMSE between observed and simulated LSWT by the FLake model under the condition of depth of the thermally active layer of bottom sediments/lake depth setting from 0.4 to 0.8 .

\begin{tabular}{cccccc}
\hline $\begin{array}{c}\text { Depth of the thermally active layer of bottom } \\
\text { sediments/lake depth }\end{array}$ & 0.4 & 0.5 & 0.6 & 0.7 & 0.8 \\
\hline & \multicolumn{5}{c}{ RMSE between simulated and } \\
observed monthly & LSWT $\left({ }^{\circ} \mathrm{C}\right)$ \\
\hline Poyang Lake & 1.19 & 1.19 & 1.19 & 1.19 & 1.19 \\
Liangzi Lake & 0.94 & 0.91 & 0.89 & 0.86 & 0.82 \\
Chao Lake & 1.54 & 1.52 & 1.50 & 1.47 & 1.45 \\
Donghu Lake & 1.28 & 1.23 & 1.20 & 1.17 & 1.14 \\
\hline
\end{tabular}

